\title{
Exponential Stability of Jump-Diffusion Systems with Neutral Term and Impulses
}

\author{
Hua Yang, ${ }^{1,2}$ Jianguo Liu, ${ }^{1}$ and Feng Jiang ${ }^{3}$ \\ ${ }^{1}$ School of Automation, Huazhong University of Science and Technology, Wuhan 430074, China \\ ${ }^{2}$ School of Mathematics and Computer Science, Wuhan Polytechnic University, Wuhan 430023, China \\ ${ }^{3}$ School of Statistics and Mathematics, Zhongnan University of Economics and Law, Wuhan 430073, China
}

Correspondence should be addressed to Feng Jiang; fjiang78@gmail.com

Received 13 January 2015; Accepted 11 February 2015

Academic Editor: Leonid Shaikhet

Copyright (C) 2015 Hua Yang et al. This is an open access article distributed under the Creative Commons Attribution License, which permits unrestricted use, distribution, and reproduction in any medium, provided the original work is properly cited.

We study jump-diffusion systems with neutral term and impulses. Under some conditions, we prove that the jump-diffusion systems with neutral term and impulses are mean square and almost surely exponentially stable. Finally, we give an example to describe the theoretical results.

\section{Introduction}

Recently, stochastic partial differential systems (SPDS) are often used to describe some evolution phenomena in studying pattern recognition and engineering $[1,2]$. Dynamic behavior of solutions for SPDS has been discussed by many researchers [3-8].

In the practical application, there exists often impulsive disturbance under specific circumstances $[9,10]$. For example, in [11, 12], Zhu et al. discussed stability behavior of stochastic impulsive systems. Sakthivel and Luo [13] discussed asymptotics of stochastic impulsive systems. Further, in [14], Jiang and Shen studied asymptotic behavior for stochastic impulsive infinite delays systems. Chen et al. [15] discussed stability of stochastic impulsive systems by inequality technique.

In addition, many models such as population models and circuits models often include the derivative terms of the current state and past state, which are often described as neutral systems [16-21]. Meanwhile, there are also a few works on jump diffusions, which are discussed extensively. For example, Zhu [22] discussed the long-time behavior of the solution including the $p$ th moment asymptotic stability and almost sure stability for stochastic jump systems. In [23, 24], the authors established dynamical behavior of stochastic jump systems and stochastic jump biological model. Cui et al.
[25-27] studied the existence, uniqueness, and some stability of stochastic jump systems. Luo and Taniguchi [28] discussed the existence of solutions of neutral stochastic jump systems under non-Lipschitz condition. Ren and Sakthivel $[29,30]$ discussed dynamic behavior of second-order jump-diffusion systems.

The rest of the paper is organized as follows. In Section 2, we give some preliminaries on mild solution. Then we give some conditions to guarantee stability of mild solution by the fixed point theory in Section 3. In Section 4, an example is presented to show our conclusions.

\section{Preliminaries}

Throughout this paper, let $\left(\Omega, \mathscr{F},\left\{\mathscr{F}_{t}\right\}_{t \geq 0}, \mathscr{P}\right)$ be a complete probability space with a filtration $\left\{\mathscr{F}_{t}\right\}_{t \geq 0}$ satisfying the usual conditions [31]. Let $\varrho>0$ and $\bar{R}_{+}=[0,+\infty)$. Moreover, let $\Xi$ and $\wp$ be real separable Hilbert spaces with norms $|\cdot|_{\Xi},|\cdot|_{\wp}$ and let $E(\wp, \Xi)$ be the space of all bounded linear operators from $\wp$ into $\Xi$. In this work, $\|\cdot\|$ is the norms of operators. The notation $\mathfrak{D}=\mathfrak{D}_{\mathscr{F}_{0}}([-\varrho, 0], \Xi)$ denotes the family of all $\mathscr{F}_{0}$-measurable functions from $[-\varrho, 0]$ into $\Xi$ with the norm $|\Psi|_{\mathscr{D}}=\sup _{t \in[-\varrho, 0]}|\Psi(t)|_{\Xi}$.

Let $\{B(t): t \geq 0\}$ be a $\wp$-valued Wiener process on the probability space $\left(\Omega, \mathscr{F},\left\{\mathscr{F}_{t}\right\}_{t \geq 0}, \mathscr{P}\right)$ with a trace class operator $Q$ on $\wp \cdot \mathscr{L}_{2}^{0}(\wp, \Xi)$ being the set of all Q-Hilbert-Schmidt 
operators from $\wp$ to $\Xi$. For the construction, the reader is referred to $[19,25,31,32]$. Assume that $m(\nu), v \in \mathfrak{D}_{m}$, is a stationary $\mathscr{F}_{v}$-Poisson point process with characteristic measure $\lambda . N(d \nu, d \vartheta)$ defined by $N(\nu, \mathbb{X})=\Sigma_{v \in \mathfrak{D}_{m}, v \geq v} 1_{\mathbb{X}}(m(v))$ for $\mathbb{X} \in \mathscr{B}(\wp-\{0\})$. Let $\widetilde{N}(d \nu, d \vartheta)=N(d \nu, d \vartheta)-\lambda(d \vartheta) d \nu$, which is independent of $B(\cdot)$. For the Poisson measure, see [21].

Suppose that $S(t), t \geq 0$, is an analytic semigroup with its infinitesimal generator $A$ [14]. For the analytic semigroup, see Pazy [32, Page 60-75]. In the paper, assume that $0 \in \rho(A)$. According to Pazy [32], a linear closed operator $(-A)^{\alpha}(\alpha \in$ $(0,1])$ can be defined on $\mathfrak{D}\left((-A)^{\alpha}\right)$.

Consider a jump-diffusion system with neutral term and impulses:

$$
\begin{aligned}
& d[Y(t)+D(t, Y(t-\kappa(t)))] \\
& =[A Y(t)+P(t, Y(t-\zeta(t)))] d t \\
& \quad+R(t, Y(t-\omega(t))) d B(t) \\
& \quad+\int_{\mathbb{X}} \Theta(t, Y(t-\theta(t)), \vartheta) \widetilde{N}(d t, d \vartheta), \quad t \neq \tau_{j}, t \geq 0, \\
& \quad \Delta Y\left(\tau_{j}\right)=H_{j}\left(Y\left(\tau_{j}^{-}\right)\right), \quad t=\tau_{j}, \quad j=1, \ldots, l,
\end{aligned}
$$

with the initial data $Y_{0}(\cdot)=\Psi \in \mathfrak{D}_{\mathscr{F}_{0}}([-\varrho, 0], \Xi)$. Here $D, P$ : $\bar{R}_{+} \times \Xi \rightarrow \Xi, R: \bar{R}_{+} \times \Xi \rightarrow \mathscr{L}_{2}^{0}(\wp, \Xi), \Theta: \bar{R}_{+} \times \Xi \times \mathbb{X} \rightarrow \Xi$ and $\kappa(t), \omega(t), \zeta(t), \theta(t): \bar{R}_{+} \rightarrow[0, \varrho]$ are continuous. Consider $H_{j}: \Xi \rightarrow \Xi, \Delta Y(v)=Y\left(v^{+}\right)-Y\left(v^{-}\right)$, where $Y\left(v^{+}\right)=$ $\lim _{\Delta v \rightarrow 0^{+}} Y(v+\Delta v)$ and $Y\left(v^{-}\right)=\lim _{\Delta v \rightarrow 0^{+}} Y(v-\Delta v), 0<$ $\tau_{1}<\cdots<\tau_{\iota}<\infty=\lim _{j \rightarrow \infty} \tau_{j}$.

Definition 1. A process $Y(t), t \in[0, T], T \in[0, \infty)$, is said to be the mild solution to system (1) if

(i) $Y(t)$ is a $\mathscr{F}_{t}$-adapted, càdlàg process and is almost surely square-integrable on $[0, T]$;

(ii) for $t \in[0, T] Y(t)$ satisfies

$$
\begin{aligned}
Y(t)= & S(t) \Psi(0)+S(t) D(0, \Psi) \\
& -D(t, Y(t-\kappa(t))) \\
& -\int_{0}^{t} A S(t-v) D(v, Y(v-\kappa(v))) d v \\
& +\int_{0}^{t} S(t-v) P(v, Y(v-\zeta(v))) d v \\
& +\int_{0}^{t} S(t-v) R(v, Y(v-\omega(v))) d B(v) \\
& +\int_{0}^{t} \int_{\mathbb{X}} S(t-v) \Theta(t, Y(v-\theta(v)), \vartheta) \widetilde{N}(d v, d \vartheta) \\
& +\sum_{0<\tau_{j}<t} S\left(t-\tau_{j}\right) H_{j}\left(Y\left(\tau_{j}^{-}\right)\right),
\end{aligned}
$$

To establish exponential stability $[7,10,20]$ of system (1), we need the following hypotheses.

$\left(H_{1}\right)\|S(t)\| \leq e^{-\beta t}$, where $\beta$ is a positive constant.

$\left(H_{2}\right)$ There exists $\widetilde{K}>0$ such that, for $t \geq 0, Y_{1}, Y_{2} \in \Xi$,

$$
\left|(-A)^{\alpha} D\left(t, Y_{1}\right)-(-A)^{\alpha} D\left(t, Y_{2}\right)\right|_{\Xi}^{2} \leq \widetilde{K}\left|Y_{1}-Y_{2}\right|_{\Xi}^{2} .
$$

$\left(H_{3}\right)$ There exist positive constants $L_{1}, L_{2}, L_{3}$ such that, for $t \geq 0, Y_{1}, Y_{2} \in \Xi$,

$$
\begin{gathered}
\left|P\left(t, Y_{1}\right)-P\left(t, Y_{2}\right)\right|_{\Xi}^{2} \leq L_{1}\left|Y_{1}-Y_{2}\right|_{\Xi}^{2}, \\
\left|R\left(t, Y_{1}\right)-R\left(t, Y_{2}\right)\right|_{\mathscr{L}_{2}^{0}}^{2} \leq L_{2}\left|Y_{1}-Y_{2}\right|_{\Xi}^{2},
\end{gathered}
$$

$\int_{\mathbb{X}}\left|\Theta\left(t, Y_{1}, \vartheta\right)-\Theta\left(t, Y_{2}, \vartheta\right)\right|_{\Xi}^{2} \lambda(d \vartheta) \leq L_{3}\left|Y_{1}-Y_{2}\right|_{\Xi}^{2}$.

$\left(H_{4}\right)$ There exist constants $l_{j}$ such that, for $Y_{1}, Y_{2} \in \Xi$, $\left|H_{j}\left(Y_{1}\right)-H_{j}\left(Y_{2}\right)\right|^{2} \leq l_{j}\left|Y_{1}-Y_{2}\right|^{2}(j=1, \ldots, l)$.

$\left(H_{5}\right)$ One has $D(t, 0)=P(t, 0)=R(t, 0)=\Theta(t, 0, \vartheta)=$ $H_{j}(0)=0(j=1,2, \ldots)$ for $t \geq 0$.

Remark 2. We should point out that it is clear that system (1) has a trivial solution when $\Psi=0$ by $\left(H_{1}\right)-\left(H_{5}\right)$.

\section{Main Results}

In the section, we will state and prove our main results on mean square and almost surely exponential stability to system (1) by the fixed point theory. To prove our main results, we firstly give a useful lemma.

Lemma 3 (see $[18,32]$ ). Under $\left(H_{1}\right)$, assume that $0 \in \rho(A)$. Then, for $\alpha \in(0,1]$,

(i) for $Y \in \mathfrak{D}\left((-A)^{\alpha}\right), S(t)(-A)^{\alpha} Y=(-A)^{\alpha} S(t) Y$;

(ii) there exist constants $M_{\alpha}>0$ and $\beta>0$ such that, for $t>0$,

$$
\left\|(-A)^{\alpha} S(t)\right\| \leq M_{\alpha} t^{-\alpha} e^{-\beta t}
$$

Now we will state and prove the main results on stability.

Theorem 4. Suppose that $\left(H_{1}\right)-\left(H_{5}\right)$ hold. Then system (1) has a unique mild solution and is mean square exponentially stable, if the initial data $\Psi$ is mean square exponentially stable and

$$
\begin{aligned}
& 6\left[\widetilde{K}\left\|(-A)^{-\alpha}\right\|^{2}+\widetilde{K} M_{1-\alpha}^{2} \beta^{-2 \alpha} \Gamma^{2}(\alpha)\right. \\
& \left.\quad+L_{1} \beta^{-2}+L_{2}(2 \beta)^{-1}+L_{3}(2 \beta)^{-1}+\widetilde{l} e^{-2 \beta T}\right]<1
\end{aligned}
$$

Here $\tilde{l}=\mathbb{E}\left(\sum_{j=1}^{\iota}\left|l_{j}\right|\right)$ and $M_{1-\alpha}$ and $\beta$ are defined by (5).

Proof. Let $\Upsilon$ be the Banach space of $Y(t)$ with the norm $\|Y\|_{\Upsilon}:=\sup _{t \geq 0} \mathbb{E}|Y(t)|_{\Xi}^{2}$ and there exist $\bar{M}>0$ and $\delta>0$ such that, for $t \geq 0$,

$$
\mathbb{E}|Y(t)|_{\Xi}^{2}<\bar{M} \mathbb{E}|\Psi|_{\mathfrak{D}}^{2} e^{-\delta t} .
$$


Define an operator $\Pi: \Upsilon \rightarrow \Upsilon$ by $\Pi(Y)(t)=\Psi(t)$ for $t \epsilon$ $[-\varrho, 0]$ and for $t \geq 0$,

$$
\begin{aligned}
\Pi(Y)(t)= & S(t) \Psi(0)+S(t) D(0, \Psi) \\
& -D(t, Y(t-\kappa(t))) \\
& -\int_{0}^{t} A S(t-v) D(v, Y(v-\kappa(v))) d v \\
& +\int_{0}^{t} S(t-v) P(v, Y(v-\zeta(v))) d v \\
& +\int_{0}^{t} S(t-v) R(v, Y(v-\varpi(v))) d B(v) \\
& +\int_{0}^{t} \int_{\mathbb{X}} S(t-v) \Theta(t, Y(v-\theta(v)), \vartheta) \widetilde{N}(d v, d \vartheta) \\
& +\sum_{0<\tau_{j}<t} S\left(t-\tau_{j}\right) H_{j}\left(Y\left(\tau_{j}^{-}\right)\right) .
\end{aligned}
$$

Now we will prove that the operator $\Pi$ has a fixed point in $\Upsilon$. Without loss of generality, we suppose that $0<\max \{\delta, \eta\}<$ $\beta$. Let $\beta_{\varsigma}:=(\beta-\varsigma)^{-1}$. We firstly claim that $\Pi(\Upsilon) \subset \Upsilon$. Let $Y(t) \in \Upsilon$ and we then have from (8)

$$
\begin{aligned}
& \mathbb{E}|\Pi(Y)(t)|_{\Xi}^{2} \\
& \leq 7 \mathbb{E}|S(t) \Psi(0)+S(t) D(0, \Psi)|_{\Xi}^{2} \\
& \quad+7 \mathbb{E}|D(t, Y(t-\kappa(t)))|_{\Xi}^{2} \\
& \quad+7 \mathbb{E}\left|\int_{0}^{t} A S(t-v) D(v, Y(v-\kappa(v))) d v\right|_{\Xi}^{2} \\
& \quad+7 \mathbb{E}\left|\int_{0}^{t} S(t-v) P(v, Y(v-\zeta(v))) d v\right|_{\Xi}^{2} \\
& \quad+7 \mathbb{E}\left|\int_{0}^{t} S(t-v) R(v, Y(v-\varpi(v))) d B(v)\right|_{\Xi}^{2} \\
& \quad+7 \mathbb{E}\left|\int_{0}^{t} \int_{X} S(t-v) \Theta(t, Y(v-\theta(v)), \vartheta) \widetilde{N}(d v, d \vartheta)\right|_{\Xi}^{2} \\
& \quad+7 \mathbb{E}\left|\sum_{0<\tau_{j}<t} S\left(t-\tau_{j}\right) H_{j}\left(Y\left(\tau_{j}^{-}\right)\right)\right|^{2} \\
& =7 \sum_{i=1}^{7} F_{i} .
\end{aligned}
$$

Note that the initial data $\Psi$ is mean square exponentially stable; that is, there exist, for $\widetilde{M}>0, \eta>0$ such that $-\varrho \leq t \leq 0, \mathbb{E}|\Psi(t)|^{2} \leq \widetilde{M} \mathbb{E}|\Psi(0)|_{\Xi}^{2} e^{-\eta t}$. By $\left(H_{2}\right)$ and $\left(H_{5}\right)$, we have

$$
\begin{aligned}
F_{2} & =\mathbb{E}\left|(-A)^{-\alpha}(-A)^{\alpha} D(t, Y(t-\kappa(t)))\right|_{\Xi}^{2} \\
& \leq \widetilde{K}\left\|(-A)^{-\alpha}\right\|^{2} \mathbb{E}|Y(t-\kappa(t))|_{\Xi}^{2} \\
& \leq \widetilde{K}\left\|(-A)^{-\alpha}\right\|^{2}\left(\bar{M} \mathbb{E}|\Psi|_{D}^{2} e^{\delta \varrho-\delta t}+\widetilde{M} \mathbb{E}|\Psi(0)|_{\Xi}^{2} e^{\eta \varrho-\eta t}\right) .
\end{aligned}
$$

Then (5) together with $\left(H_{2}\right)$ and $\left(H_{5}\right)$ yields

$$
\begin{aligned}
F_{3} \leq & M_{1-\alpha}^{2} \mathbb{E}\left(\int_{0}^{t} t_{v}^{(1-\alpha) / 2} e^{-\beta(t-v) / 2} t_{v}^{(1-\alpha) / 2} e^{-\beta(t-v) / 2}\right. \\
\cdot & \left.\left|(-A)^{\alpha} D(v, Y(v-\kappa(v)))\right|_{\Xi} d v\right)^{2} \\
\leq & M_{1-\alpha}^{2} \int_{0}^{t} e^{\beta v-\beta t} t_{v}^{1-\alpha} d v \\
& \cdot \int_{0}^{t} e^{\beta v-\beta t} t_{v}^{1-\alpha} \mathbb{E}\left|(-A)^{\alpha} D(v, Y(v-\kappa(v)))\right|_{\Xi}^{2} d v \\
\leq & \widetilde{K} \Gamma(\alpha) \beta^{-\alpha} M_{1-\alpha}^{2} \\
& \cdot \int_{0}^{t} e^{\beta v-\beta t} t_{v}^{1-\alpha} \mathbb{E}|Y(v-\kappa(v))|_{\Xi}^{2} d v \\
\leq & \widetilde{K} \Gamma(\alpha) \beta^{-\alpha} M_{1-\alpha}^{2} \\
& \cdot \int_{0}^{t} e^{\beta v-\beta t} t_{v}^{1-\alpha} \\
& \cdot\left(\bar{M} \mathbb{E}|\Psi|_{\mathscr{D}}^{2} e^{\delta Q-\delta v}+\widetilde{M} \mathbb{E}|\Psi(0)|_{\Xi}^{2} e^{\eta \varrho-\eta v}\right) d v \\
\leq & \widetilde{K} \Gamma^{2}(\alpha) \beta^{-\alpha} M_{1-\alpha}^{2} \\
& \cdot\left(\beta_{\delta}^{\alpha} \bar{M} \mathbb{E}|\Psi|_{\mathscr{D}}^{2} e^{\delta Q-\delta t}+\beta_{\eta}^{\alpha} \widetilde{M} \mathbb{E}|\Psi|_{\mathscr{D}}^{2} e^{\eta \varrho-\eta t}\right) .
\end{aligned}
$$

By $\left(H_{1}\right)$, we have

$$
\begin{aligned}
F_{4} \leq & \mathbb{E}\left(\int_{0}^{t} e^{\beta v-\beta t}|P(v, Y(v-\zeta(v)))|_{\Xi} d v\right)^{2} \\
\leq & \int_{0}^{t}\left(e^{(\beta v-\beta t) / 2}\right)^{2} d v \\
& \cdot \mathbb{E}\left(\int^{t}\left(e^{(\beta v-\beta t) / 2}|P(v, Y(v-\zeta(v)))|_{\Xi}\right)^{2} d v\right) \\
\leq & \frac{L_{1}}{\beta} \int_{0}^{t} e^{\beta v-\beta t} \mathbb{E}|Y(v-\zeta(v))|_{\Xi}^{2} d v \\
\leq & \frac{L_{1}}{\beta}\left(\beta_{\delta} \bar{M} \mathbb{E}|\Psi|_{\mathscr{D}}^{2} e^{\delta \varrho-\delta t}+\beta_{\eta} \widetilde{M} \mathbb{E}|\Psi|_{\mathscr{D}}^{2} e^{\eta \varrho-\eta t}\right) .
\end{aligned}
$$


By the properties of the martingales, we have

$$
\begin{aligned}
F_{5} & \leq \int_{0}^{t} \mathbb{E}|S(v-t) R(v, Y(v-\Phi(v)))|_{\mathscr{L}_{2}^{0}}^{2} d v \\
& \leq \int_{0}^{t} e^{2 \beta v-2 \beta t} \mathbb{E}|R(v, Y(v-\Phi(v)))|_{\mathscr{L}_{2}^{0}}^{2} d v \\
& \leq L_{2} \int_{0}^{t} e^{2 \beta v-2 \beta t} \mathbb{E}|Y(v-\omega(v))|_{\Xi}^{2} d v \\
& \leq L_{2} \int_{0}^{t} e^{2 \beta v-2 \beta t}\left(\bar{M} \mathbb{E}|\Psi|_{\mathfrak{D}}^{2} e^{\delta \varrho-\delta v}+\widetilde{M} \mathbb{E}|\Psi|_{\Xi}^{2} e^{\eta \varrho-\eta v}\right) d v \\
& \leq L_{2}\left(\beta_{\delta} \bar{M} \mathbb{E}|\Psi|_{\mathfrak{D}}^{2} e^{2 \delta \varrho-2 \delta t}+\beta_{\eta} \widetilde{M} \mathbb{E}|\Psi|_{\Xi}^{2} e^{\eta \varrho-\eta t}\right), \\
F_{6} & \leq \mathbb{E} \int_{0}^{t} \int_{\mathbb{X}} e^{2 \beta v-2 \beta t}|\Theta(v, Y(v-\theta(v)), \vartheta)|^{2} \lambda(d \vartheta) d v \\
& \leq L_{3} \int_{0}^{t} e^{2 \beta v-2 \beta t} \mathbb{E}|Y(v-\theta(v))|_{\Xi}^{2} d v \\
& \leq L_{3}\left(\beta_{\delta} \bar{M} \mathbb{E}|\Psi|_{\mathfrak{D}}^{2} e^{\delta \varrho-\delta t}+\beta_{\eta} \widetilde{M} \mathbb{E}|\Psi|_{\Xi}^{2} e^{\eta Q^{-\eta} t}\right) .
\end{aligned}
$$

By $\left(H_{1}\right)$ and $\left(H_{4}\right)$, we have

$$
F_{7} \leq e^{-2 \beta t} l_{j} \mathbb{E}\left|Y\left(t_{j}^{-}\right)\right|_{\Xi}^{2} .
$$

From (9) to (14), we can see obviously that there exist $\widehat{M}>0$ and $\widehat{\delta}>0$ such that

$$
\mathbb{E}|\Pi(Y)(t)|_{\Xi}^{2} \leq \widehat{M} \mathbb{E}|\Psi|_{\mathfrak{D}}^{2} e^{-\widehat{\delta} t}
$$

Next we claim that $\Pi(Y)(t)$ is càdlàg on $\Upsilon$. Let $Y \in \Upsilon, \widehat{t} \geq 0$, and $\Delta \widehat{t}>0$; we have from (8) that

$$
\begin{aligned}
& \mathbb{E}|\Pi(Y)(\widehat{t}+\Delta \widehat{t})-\Pi(Y)(\widehat{t})|_{\Xi}^{2} \\
& \quad \leq 7 \sum_{i=1}^{7} \mathbb{E}\left|F_{i}(\widehat{t}+\Delta \widehat{t})-F_{i}(\widehat{t})\right|_{\Xi}^{2} .
\end{aligned}
$$

We can easily see that $\mathbb{E}\left|F_{i}(\hat{t}+\Delta \hat{t})-F_{i}(\hat{t})\right|_{\Xi}^{2} \rightarrow 0$ as $\Delta \widehat{t} \rightarrow 0$, $i=1, \ldots, 4$, and $i=7$. Moreover, by the properties of the martingales, we have the fact that when $\Delta \widehat{t} \rightarrow 0$,

$$
\begin{aligned}
& \mathbb{E}\left|F_{5}(\widehat{t}+\Delta \hat{t})-F_{5}(\hat{t})\right|_{\Xi}^{2} \\
& \leq 2 \int_{0}^{\widehat{t}} \mathbb{E} \mid(S(\widehat{t}+\Delta \widehat{t}-v)-S(\widehat{t}-v)) \\
& \left.\quad \cdot R(v, Y(v-\zeta(v)))\right|_{\Xi} ^{2} d v \\
& +2 \int_{\widehat{t}}^{\widehat{t}+\Delta \widehat{t}} \mathbb{E}|S(\widehat{t}+\Delta \widehat{t}-v) R(v, Y(v-\zeta(v)))|_{\Xi}^{2} d v \\
& \longrightarrow 0,
\end{aligned}
$$

$$
\begin{aligned}
& \mathbb{E}\left|F_{6}(\widehat{t}+\Delta \widehat{t})-F_{6}(\widehat{t})\right|_{\Xi}^{2} \\
& \leq 2 \mathbb{E} \mid \int_{0}^{\widehat{t}} \int_{\mathbb{X}}(S(\widehat{t}+\Delta \widehat{t}-v)-S(\widehat{t}-v) \\
& \left.\cdot \Theta(t, Y(v-\theta(v)), \vartheta) \widetilde{N}(d v, d \vartheta)\right|_{\Xi} ^{2} \\
& +2 \mathbb{E} \mid \int_{\widehat{t}}^{\widehat{t}+\Delta \widehat{t}} \int_{\mathbb{X}} S(\widehat{t}+\Delta \widehat{t}-v) \\
& \leq 2\|S(\Delta \widehat{t})-I\|^{2} \mathbb{E} \\
& \quad \cdot \int_{0}^{\hat{t}} \int_{\mathbb{X}}|S(\widehat{t}-v) \Theta(t, Y(v-\theta(v)), \vartheta)|_{\Xi}^{2} v(d \vartheta) d v \\
& +\left.2 \mathbb{E} \quad \cdot \Theta(t, Y(v-\theta(v)), \vartheta) \widetilde{N}(d v, d \vartheta)\right|_{\Xi} ^{2} \\
& \quad \cdot \int_{\widehat{t}}^{\hat{t}+\Delta \hat{t}} \int_{\mathbb{X}}|S(\widehat{t}+\Delta \widehat{t}-v) \Theta(t, Y(v-\theta(v)), \vartheta)|_{\Xi}^{2} \\
& +v(d \vartheta) d v
\end{aligned}
$$$$
\longrightarrow 0 \text {. }
$$

Consequently, we obtain that $\Pi(\Upsilon) \subset \Upsilon$.

We finally claim $\Pi$ is contractive. From (8), $Y_{1}, Y_{2} \in \Upsilon$,

$$
\sup _{0 \leq t \leq T} \mathbb{E}\left|\Pi\left(Y_{1}\right)(t)-\Pi\left(Y_{2}\right)(t)\right|_{\Xi}^{2} \leq 6 \sum_{i=1}^{6} G_{i} .
$$

Similar to (10)-(14), we have

$$
\begin{aligned}
& G_{1}=\sup _{0 \leq t \leq T} \mathbb{E}\left|D\left(t, Y_{1}(t-\kappa(t))\right)-D\left(t, Y_{2}(t-\kappa(t))\right)\right|_{\Xi}^{2} \\
& \leq \widetilde{K}\left\|(-A)^{-\alpha}\right\|^{2} \sup _{0 \leq v \leq T} \mathbb{E}\left|Y_{1}(v)-Y_{2}(v)\right|_{\Xi}^{2}, \\
& G_{2}=\sup _{0 \leq t \leq T} \mathbb{E} \mid \int_{0}^{t} A S(t-v) \\
& \cdot\left[D\left(v, Y_{1}(v-\kappa(v))\right)\right. \\
& \left.-D\left(v, Y_{2}(v-\kappa(v))\right)\right]\left.d v\right|_{\Xi} ^{2} \\
& \leq \widetilde{K} M_{1-\alpha}^{2} \beta^{-2 \alpha} \Gamma^{2}(\alpha) \sup _{0 \leq v \leq T} \mathbb{E}\left|Y_{1}(v)-Y_{2}(v)\right|_{\Xi}^{2}, \\
& G_{3}=\sup _{0 \leq t \leq T} \mathbb{E} \mid \int_{0}^{t} S(t-v) \\
& \cdot\left[P\left(v, Y_{1}(v-\zeta(v))\right)\right. \\
& \left.-P\left(v, Y_{2}(v-\zeta(v))\right)\right]\left.d v\right|_{\Xi} ^{2} \\
& \leq L_{1} \beta^{-2} \sup _{0 \leq v \leq T} \mathbb{E}\left|Y_{1}(v)-Y_{2}(v)\right|_{\Xi}^{2},
\end{aligned}
$$




$$
\begin{aligned}
& G_{4}=\sup _{0 \leq t \leq T} \mathbb{E} \mid \int_{0}^{t} S(t-v) \\
& \cdot\left[R\left(v, Y_{1}(v-\omega(v))\right)\right. \\
& \left.-R\left(v, Y_{2}(v-\omega(v))\right)\right]\left.d B(v)\right|_{\Xi} ^{2} \\
& \leq L_{2}(2 \beta)^{-1} \sup _{0 \leq v \leq T} \mathbb{E}\left|Y_{1}(v)-Y_{2}(v)\right|_{\Xi}^{2}, \\
& G_{5}=\sup _{0 \leq t \leq T} \mathbb{E} \mid \int_{0}^{t} \int_{\mathbb{X}} S(v-t) \\
& \cdot\left[\Theta\left(t, Y_{1}(v-\theta(v)), \vartheta\right)\right. \\
& \left.-\Theta\left(t, Y_{2}(v-\theta(v)), \vartheta\right)\right] \\
& \left.\widetilde{N}(d v, d \vartheta)\right|_{\Xi} ^{2} \\
& \leq L_{3}(2 \beta)^{-1} \sup _{0 \leq v \leq T} \mathbb{E}\left|Y_{1}(v)-Y_{2}(v)\right|_{\Xi}^{2}, \\
& G_{6}=\sup _{0 \leq t \leq T} \mathbb{E}\left|\sum_{0<\tau_{j}<t} S\left(t-\tau_{j}\right)\left(H_{j}\left(Y_{1}\left(\tau_{j}^{-}\right)\right)-H_{j}\left(Y_{2}\left(\tau_{j}^{-}\right)\right)\right)\right|^{2} \\
& \leq \tilde{l} e^{-2 \beta T} \sup _{0 \leq v \leq T} \mathbb{E}\left|Y_{1}(v)-Y_{2}(v)\right|_{\Xi}^{2} .
\end{aligned}
$$

Here $\widetilde{l}=\mathbb{E}\left(\sum_{j=1}^{l}\left|l_{j}\right|\right)$.

Consequently, we have

$$
\begin{gathered}
\sup _{0 \leq t \leq T} \mathbb{E}\left|\Pi\left(Y_{1}\right)(t)-\Pi\left(Y_{2}\right)(t)\right|_{\Xi}^{2} \\
\leq 6\left[\widetilde{K}\left\|(-A)^{-\alpha}\right\|^{2}+\widetilde{K} M_{1-\alpha}^{2} \beta^{-2 \alpha} \Gamma^{2}(\alpha)\right. \\
+L_{1} \beta^{-2}+L_{2}(2 \beta)^{-1} \\
\left.+L_{3}(2 \beta)^{-1}+\widetilde{l} e^{-2 \beta T}\right] \\
\cdot \sup _{0 \leq v \leq T} \mathbb{E}\left|Y_{1}(v)-Y_{2}(v)\right|_{\Xi}^{2} .
\end{gathered}
$$

Then if (6) holds, $\Pi$ is contractive. Therefore, system (1) has a unique $Y(t) \in \Upsilon$ and $Y(t)$ is mean square exponentially stable if (6) holds. This proof is complete.

According to [5], we similarly have the following.

Theorem 5. Under the conditions in Theorem 4, system (1) is almost surely exponentially stable.
If $\Theta=0$, system (1) becomes

$$
\begin{aligned}
& d[Y(t)+D(t, Y(t-\kappa(t)))] \\
& =[A Y(t)+P(t, Y(t-\zeta(t)))] d t \\
& +R(t, Y(t-\omega(t))) d B(t), \quad t \geq 0, \quad t \neq \tau_{j}, \\
& \Delta Y\left(\tau_{j}\right)=H_{j}\left(Y\left(\tau_{j}^{-}\right)\right), \quad t=\tau_{j}, j=1, \ldots, l,
\end{aligned}
$$

with the initial data $Y_{0}(\cdot)=\Psi \in \mathfrak{D}_{\mathscr{F}_{0}}([-\varrho, 0], \Xi)$.

From Theorems 4 and 5, we have the following.

Corollary 6. Assume that the conditions in Theorem 4 hold, but (6) is replaced with the following condition:

$$
\begin{aligned}
& 5\left[\widetilde{K}\left\|(-A)^{-\alpha}\right\|^{2}+M_{1-\alpha}^{2} \widetilde{K} \beta^{-2 \alpha} \Gamma^{2}(\alpha)\right. \\
& \left.\quad+L_{1} \beta^{-2}+L_{2}(2 \beta)^{-1}+e^{-2 \beta T} \mathbb{E}\left(\sum_{j=1}^{l}\left|l_{j}\right|\right)\right]<1 .
\end{aligned}
$$

Then system (22) admits a unique mild solution and is mean square and almost surely exponentially stable.

$$
\begin{aligned}
& \text { If } \Theta \equiv 0 \text { and } D \equiv 0 \text {, system }(1) \text { becomes } \\
& \begin{aligned}
d Y(t)= & {[A Y(t)+P(t, Y(t-\zeta(t)))] d t } \\
& +R(t, Y(t-\omega(t))) d B(t), \quad t \geq 0, t \neq \tau_{j},
\end{aligned} \\
& \qquad Y\left(\tau_{j}\right)=H_{j}\left(Y\left(\tau_{j}^{-}\right)\right), \quad t=\tau_{j}, \quad j=1, \ldots, l,
\end{aligned}
$$

with the initial data $Y_{0}(\cdot)=\Psi \in \mathfrak{D}_{\mathscr{F}_{0}}([-\varrho, 0], \Xi)$.

Corollary 7. Assume that the conditions in Theorem 4 hold, but $\left(\mathrm{H}_{2}\right)$ and (6) are replaced with the following condition:

$$
4\left(L_{1} \beta^{-2}+L_{2}(2 \beta)^{-1}+e^{-2 \beta T} \mathbb{E}\left(\sum_{j=1}^{\iota}\left|l_{j}\right|\right)\right)<1 .
$$

Then system (24) has a unique mild solution and is mean square and almost surely exponentially stable.

Remark 8. We think that the results of the paper can be generalized to infinite delay systems. Systems (22) and (24) have been discussed in [14] and [13], respectively, which focus on asymptotic stability of mild solution. Also by Theorem 4 system (1) without impulses is also mean square and almost surely exponential stability under some conditions, which has been studied in [25]. However, it is well known that there are great differences on the method between the time-delay cases, in particular when considering a problem involved in perturbation. In the paper, we mainly focus on exponential stability. In the sense, $[13,14,25]$ are generalized to more extensive systems.

Remark 9. In particular, when $D \equiv 0, \Theta \equiv 0$, system (1) without jumps, impulses, and neutral term reduces to SPDS, 
which is mean square and almost surely exponential stability if $3\left(L_{1} \beta^{-2}+L_{2}(2 \beta)^{-1}\right)<1$. When $L_{1} \beta^{-2}+L_{2} \beta^{-1}<1 / 3$, Luo [5] showed that system (1) without jumps, impulses, and neutral term is mean square exponentially stable to this system. In the sense, the result of the paper improves the result of [5].

Remark 10. Besides, it should be pointed out that the proposed method in the paper can be employed to consider the $p$ th moment $(p \geq 2)$ exponential stability to system (1).

\section{Illustrative Example}

Example 1. Consider a jump-diffusion system with neutral term and impulses:

$$
\begin{aligned}
& d\left(Y(t, \chi)+\beta_{1} Y(t-\kappa(t), \chi)\right) \\
& =\left(\frac{\partial^{2}}{\partial \chi^{2}} Y(t, \chi)+\beta_{2} Y(t-\zeta(t), \chi)\right) d t \\
& \quad+\beta_{3} Y(t-\omega(t), \chi) d B(t) \\
& \quad+\int_{X} \beta_{4} \vartheta Y(t-\theta(t), \chi) \widetilde{N}(d t, d \vartheta), \quad t \geq 0, \\
& \Delta Y\left(\tau_{j}, \chi\right)=b_{j} Y\left(\tau_{j}^{-}, \chi\right), \quad t=\tau_{j}(j=1,2,3, \ldots, \iota),
\end{aligned}
$$

with $Y(s, \cdot)=\Psi(s, \cdot) \in \mathscr{L}^{2}[0, \pi], Y(\cdot, 0)=Y(\cdot, \pi)=0, s \leq 0$, where $\beta_{j}>0, b_{j} \geq 0$ and $\sum_{j=1}^{l} b_{j}<\infty$.

Let $\mathbb{X}=\{\mathfrak{x} \in R: 0<|\mathfrak{F}| \leq \ell, \ell>0\}$ and $\mathfrak{H}=L^{2}(0, \pi)$. The operator $A$ is defined by $A: \mathfrak{H} \rightarrow \mathfrak{H}$ with $A=\partial^{2} / \partial \chi^{2}$ and

$$
\begin{gathered}
\mathfrak{D}(A)=\left\{Y \in \mathfrak{H}: Y, \frac{\partial Y}{\partial \chi}\right. \text { are absolutely continuous, } \\
\left.\frac{\partial^{2} Y}{\partial \chi^{2}} \in \mathfrak{H}, Y(0)=Y(\pi)=0\right\} ;
\end{gathered}
$$

then $(-A)^{3 / 5}$ is given by

$$
(-A)^{3 / 5} Y=\sum_{n=1}^{\infty} n\left\langle Y, \sqrt{\frac{2}{n}} \sin n \chi\right\rangle_{\mathfrak{H}} \sqrt{\frac{2}{n}} \sin n \chi,
$$

and the domain

$$
\begin{aligned}
& \mathfrak{D}\left((-A)^{3 / 5}\right) \\
& \quad=\left\{Y \in \mathfrak{H}, \sum_{n=1}^{\infty} n\left\langle Y, \sqrt{\frac{2}{n}} \sin n \chi\right\rangle_{\mathfrak{S}} \sqrt{\frac{2}{n}} \sin n \chi \in \mathfrak{H}\right\} .
\end{aligned}
$$

Since, for $t \geq 0,\|S(t)\| \leq \exp \left(-\pi^{2} t\right)$, from Pazy [32, Page 70], we have

$$
\left\|(-A)^{-3 / 5}\right\| \leq \frac{1}{\Gamma(3 / 5)} \int_{0}^{\infty} v^{-2 / 5}\|S(v)\| d v \leq \frac{1}{\pi^{6 / 5}} .
$$

Obviously, $\left(H_{1}\right)-\left(H_{5}\right)$ are satisfied with

$$
\begin{aligned}
& \beta=\pi^{2}, \quad \widetilde{K}=\left\|(-A)^{3 / 5}\right\|^{2} \beta_{1}^{2}, \quad L_{1}=\beta_{2}^{2}, \\
& L_{2}=\beta_{3}^{2}, \quad L_{3}=\beta_{4}^{2} \int_{X} \vartheta^{2} \lambda(d \vartheta), \quad l_{j}=b_{j}^{2} \text {. }
\end{aligned}
$$

Thus, by Theorems 4 and 5, system (26) is mean square and almost surely exponentially stable if

$$
\begin{aligned}
\left\|(-A)^{3 / 5}\right\|^{2} \beta_{1}^{2}\left(\frac{1}{\pi^{2}}+\frac{M_{2 / 5}^{2}}{\pi}\right)+\frac{\beta_{2}^{2}}{\pi^{4}}+\frac{\beta_{3}^{2}}{2 \pi^{2}} \\
+\frac{\beta_{4}^{2}}{2 \pi^{2}} \int_{\mathbb{X}} \vartheta^{2} \lambda(d \vartheta)+e^{-2 \pi T} \mathbb{E}\left(\sum_{j=1}^{l}\left|b_{j}\right|^{2}\right)<\frac{1}{6},
\end{aligned}
$$

where $M_{2 / 5}$ is defined by (5).

\section{Concluding Remarks}

In this paper, we have discussed jump-diffusion systems with neutral term and impulses. Some conditions on mean square and almost surely exponential stability of the mild solutions to the jump-diffusion systems with neutral term and impulses are derived by the fixed point theory. The obtained results extend some earlier results to the case of SPDS with neutral term and jump and impulses. Finally, the results of this paper are demonstrated well with an example.

\section{Conflict of Interests}

The authors declare that there is no conflict of interests regarding the publication of this paper.

\section{Acknowledgments}

The work is supported by the National Natural Science Foundation of China (61304067 and 61071136), the Natural Science Foundation of Hubei Province of China (2013CFB443), and Research Fund for the Doctoral Program of Higher Education of China (20110142110069).

\section{References}

[1] J. Wu, Theory and Applications of Partial Functional Differential Equations, vol. 119 of Applied Mathematical Sciences, Springer, New York, NY, USA, 1996.

[2] K. Liu, Stability of Infinite Dimensional Stochastic Differential Equations with Applications, Chapman \& Hall, CRC Press, London, UK, 2006.

[3] G. da Prato and J. Zabczyk, Stochastic Equations in Infinite Dimensions, vol. 44 of Encyclopedia of Mathematics and its Applications, Cambridge University Press, Cambridge, UK, 1992.

[4] T. Caraballo and K. Liu, "Exponential stability of mild solutions of stochastic partial differential equations with delays," Stochastic Analysis and Applications, vol. 17, no. 5, pp. 743-763, 1999. 
[5] J. Luo, "Fixed points and exponential stability of mild solutions of stochastic partial differential equations with delays," Journal of Mathematical Analysis and Applications, vol. 342, no. 2, pp. 753-760, 2008.

[6] J. Luo and T. Taniguchi, "Fixed points and stability of stochastic neutral partial differential equations with infinite delays," Stochastic Analysis and Applications, vol. 27, no. 6, pp. 1163-1173, 2009.

[7] H. Chen, "Integral inequality and exponential stability for neutral stochastic partial differential equations with delays," Journal of Inequalities and Applications, vol. 2009, no. 1, Article ID 297478, 2009.

[8] L. Pan, "Local stability and parameter dependence of mild solutions for stochastic differential equations," Advances in Difference Equations, vol. 2014, no. 1, article 276, 2014.

[9] M. Benchohra, J. Henderson, and S. Ntouyas, Impulsive Differential Equations and Inclusions, Hindawi Publishing Corporation, New York, NY, USA, 2006.

[10] L. Zhang, Y. Ding, T. Wang, L. Hu, and K. Hao, "Stability of neutral impulsive nonlinear stochastic evolution equations with time varying delays," Asian Journal of Control, vol. 16, no. 5, pp. 1416-1424, 2014.

[11] Q. Zhu and B. Song, "Exponential stability of impulsive nonlinear stochastic differential equations with mixed delays," Nonlinear Analysis: Real World Applications, vol. 12, no. 5, pp. 2851-2860, 2011.

[12] Q. Zhu and J. Cao, "Stability analysis of markovian jump stochastic BAM neural networks with impulse control and mixed time delays," IEEE Transactions on Neural Networks and Learning Systems, vol. 23, no. 3, pp. 467-479, 2012.

[13] R. Sakthivel and J. Luo, "Asymptotic stability of nonlinear impulsive stochastic differential equations," Statistics \& Probability Letters, vol. 79, no. 9, pp. 1219-1223, 2009.

[14] F. Jiang and Y. Shen, "Stability of impulsive stochastic neutral partial differential equations with infinite delays," Asian Journal of Control, vol. 14, no. 6, pp. 1706-1709, 2012.

[15] H. Chen, C. Zhu, and Y. Zhang, "A note on exponential stability for impulsive neutral stochastic partial functional differential equations," Applied Mathematics and Computation, vol. 227, pp. 139-147, 2014.

[16] F. Jiang, "Mild solutions of neutral semilinear stochastic functional dynamic systems with local non-Lipschitz coefficients," Advances in Mathematical Physics, vol. 2013, Article ID 823535, 6 pages, 2013.

[17] J. Bao and Z. Hou, "Existence of mild solutions to stochastic neutral partial functional differential equations with nonLipschitz coefficients," Computers \& Mathematics with Applications, vol. 59, no. 1, pp. 207-214, 2010.

[18] F. Jiang and Y. Shen, "A note on the existence and uniqueness of mild solutions to neutral stochastic partial functional differential equations with non-Lipschitz coefficients," Computers \& Mathematics with Applications, vol. 61, no. 6, pp. 1590-1594, 2011.

[19] H. Bao and J. Cao, "Existence and uniqueness of solutions to neutral stochastic functional differential equations with infinite delay," Applied Mathematics and Computation, vol. 215, no. 5, pp. 1732-1743, 2009.

[20] T. E. Govindan, "Almost sure exponential stability for stochastic neutral partial functional differential equations," Stochastics, vol. 77, no. 2, pp. 139-154, 2005.
[21] J. Bao, A. Truman, and C. Yuan, "Stability in distribution of mild solutions to stochastic partial differential delay equations with jumps," Proceedings of The Royal Society of London. Series A. Mathematical, Physical and Engineering Sciences, vol. 465, no. 2107, pp. 2111-2134, 2009.

[22] Q. Zhu, "Asymptotic stability in the pth moment for stochastic differential equations with Lévy noise," Journal of Mathematical Analysis and Applications, vol. 416, no. 1, pp. 126-142, 2014.

[23] X. Yang and Q. Zhu, "pth moment exponential stability of stochastic partial differential equations with Poisson jumps," Asian Journal of Control, vol. 16, no. 5, pp. 1482-1491, 2014.

[24] L. Guo and Q. Zhu, "Stability analysis for stochastic VolterraLevin equations with Poisson jumps: fixed point approach," Journal of Mathematical Physics, vol. 52, no. 4, Article ID 042702, 15 pages, 2011.

[25] J. Cui, L. Yan, and X. Sun, "Exponential stability for neutral stochastic partial differential equations with delays and Poisson jumps," Statistics \& Probability Letters, vol. 81, no. 12, pp. 19701977, 2011.

[26] J. Cui and L. Yan, "Successive approximation of neutral stochastic evolution equations with infinite delay and Poisson jumps," Applied Mathematics and Computation, vol. 218, no. 12, pp. 6776-6784, 2012.

[27] J. Cui and L. Yan, "Existence result for fractional neutral stochastic integro-differential equations with infinite delay," Journal of Physics. A. Mathematical and Theoretical, vol. 44, no. 33, Article ID 335201, 2011.

[28] J. Luo and T. Taniguchi, “The existence and uniqueness for nonLipschitz stochastic neutral delay evolution equations driven by Poisson jumps," Stochastics and Dynamics, vol. 9, no. 1, pp. 135152, 2009.

[29] Y. Ren and R. Sakthivel, "Existence, uniqueness, and stability of mild solutions for second-order neutral stochastic evolution equations with infinite delay and Poisson jumps," Journal of Mathematical Physics, vol. 53, no. 7, Article ID 073517, 2012.

[30] R. Sakthivel and Y. Ren, "Exponential stability of second-order stochastic evolution equations with Poisson jumps," Communications in Nonlinear Science and Numerical Simulation, vol. 17, no. 12, pp. 4517-4523, 2012.

[31] H. Yang and F. Jiang, "Exponential stability of mild solutions to impulsive stochastic neutral partial differential equations with memory," Advances in Difference Equations, vol. 2013, article 148, 2013.

[32] A. Pazy, Semigroups of Linear Operators and Applications to Partial Differential Equations, Springer, New York, NY, USA, 1992. 


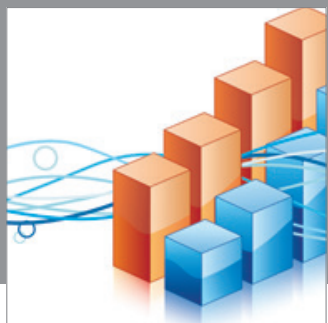

Advances in

Operations Research

mansans

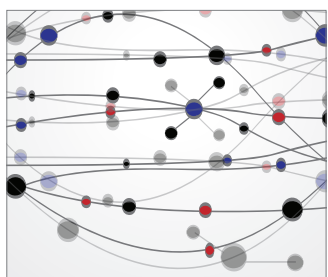

The Scientific World Journal
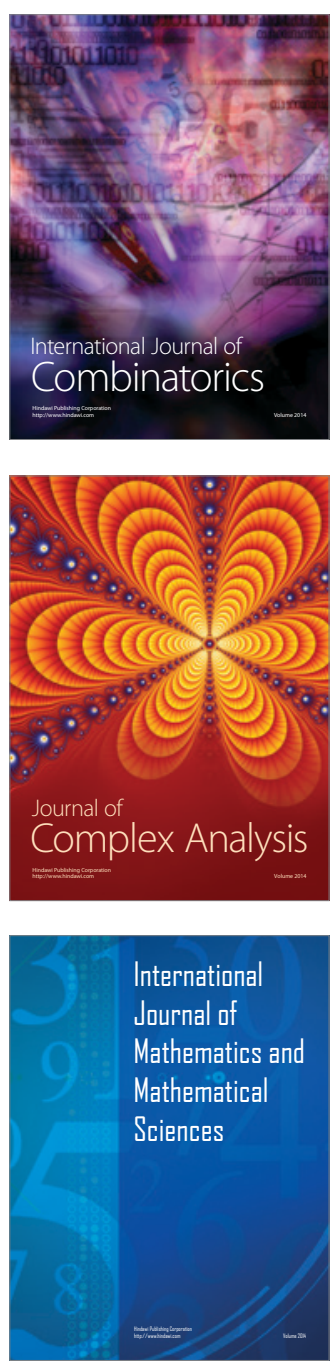
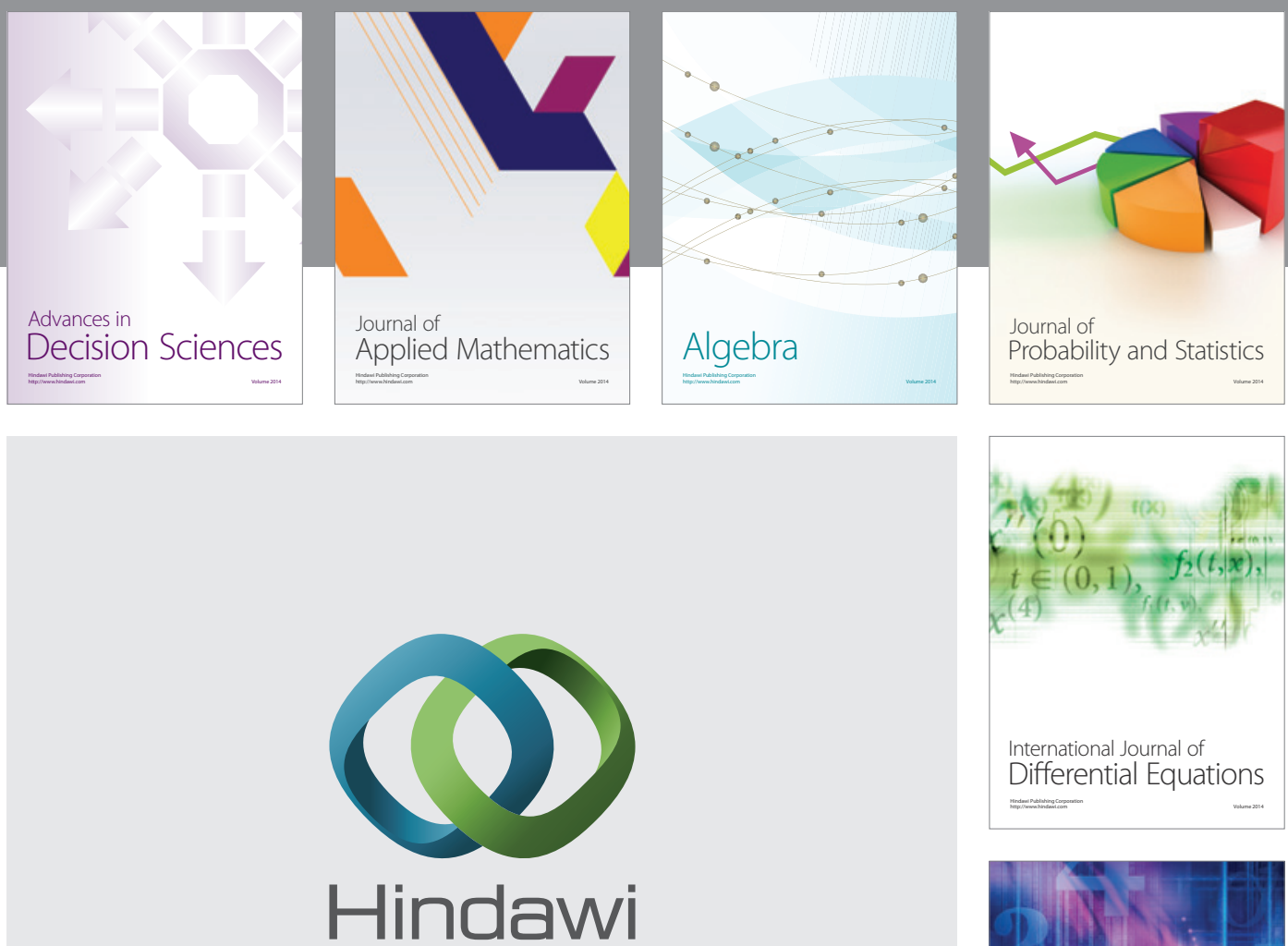

Submit your manuscripts at http://www.hindawi.com
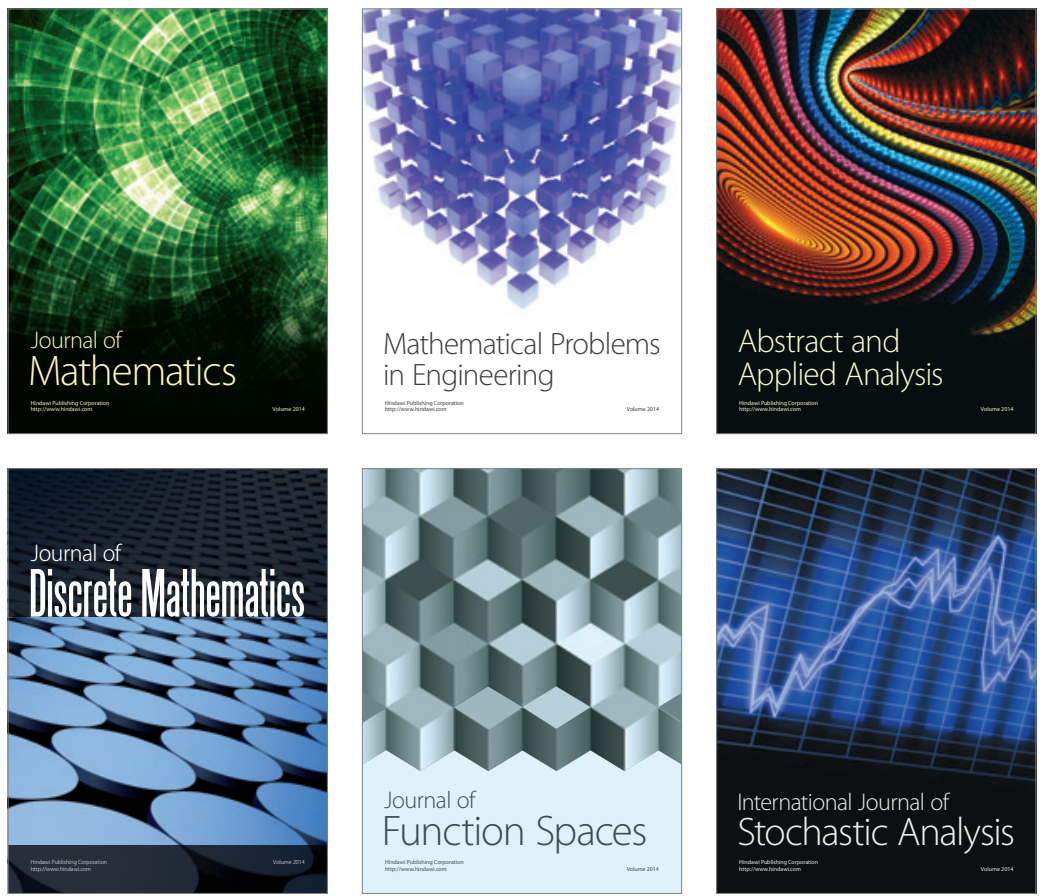

Journal of

Function Spaces

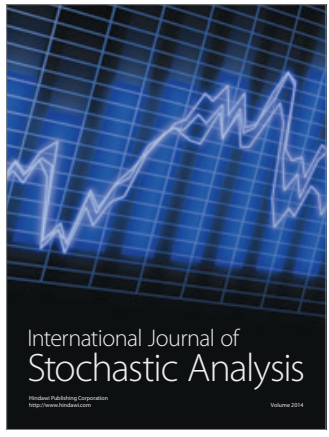

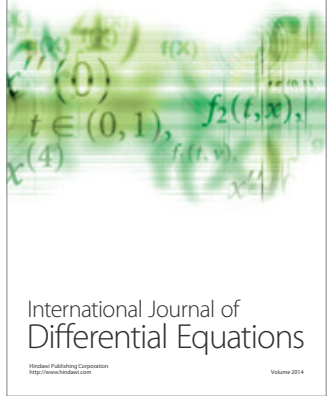
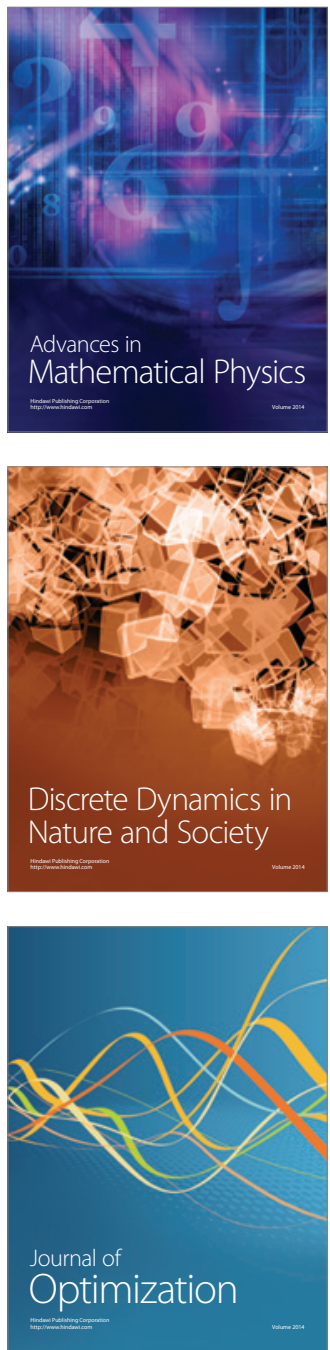